\title{
Factors Predicting Emotional Cue-Responding Behaviors of Nurses in Taiwan: An Observational Study
}

Mei-Feng Lin, An-Yu Lee, Cheng-Chen Chou, Tien-Yu Liu, Chia-Chun Tang

This is the author's manuscript of the article published in final edited form as:

Lin, M.-F., Lee, A.-Y., Chou, C.-C., Liu, T.-Y., \& Tang, C.-C. (2016). Factors predicting emotional cue-responding behaviors of nurses in Taiwan: An observational study. Psycho-Oncology, n/a-n/a. https://doi.org/10.1002/pon.4330 


\section{Background}

According to the World Health Organization 2012 GLOBOCAN project, more than 32 million people have suffered from cancer worldwide [1]. In addition to physical symptoms, cancer brings profound psychological impacts. Based on two studies examining thousands of cancer patients, the prevalence rate of psychological distress was between 32-44\% [2, 3]. In Asia, a systematical review $(n=43)$ showed that approximately one third of prostate cancer patients experienced clinically high psychological distress [4]. Moreover, evidence indicated that more than a quarter of Asian cancer patients experienced depression and anxiety [5-7]. This emotional distress is negatively linked to health-related quality of life, emotional function, role function, and social function [8].

\section{Patients’ Expression of Emotional Distress: Emotional Cues}

To detect patients' emotional distress, understanding how patients express their emotions is essential. Heaven and Green (2001) conducted a series of relative studies and conceptualized these expressions as emotional cues. An emotional cue is "a hint or a clear expression that something is or may be important or distressing or a cause of concern” [9]. Emotional cues are reliable indicators of emotional distress. Patients with emotional distress were more likely to express emotional cues in consultations than were those without emotional distress [8].

Researchers identified special patterns of how cancer patients express their emotional cues. A study revealed that cancer patients $(n=196)$ expressed more emotional cues earlier in the provider-patient conversation than later. Moreover, patients expressed more emotional cues in their conversation with nurses than with physicians [10]. Clearly, nurses play an important role in responding to patients' emotional cues. Appropriate nursing responses to a patient's emotional cues not only enhance the patient's information recall and satisfaction, but also predict nurses' own satisfaction [11]. Evidences suggested that nurses' sufficient competence in responding to emotional cues (CRE) encourages further disclosure, and is the foundation of providing emotionfocused care [12-15].

\section{Patient-related Factors Influencing Emotional Cues Disclosure}

Healthcare providers face several challenges to recognizing and responding appropriately to patients' emotional cues. Patients may hide or blur their emotional cues from nurses for several reasons, including worrying about treatment delay, fear of the stigma of being a difficult patient, trying to be “cooperative,” or searching for emotional support from family only [16]. Approximately 70\% of cancer patients' emotional cues were implicit and were therefore hard to detect by Dutch nurses in one study [13]. In an American study, when 57\% of cancer patients' emotional cues were acknowledged, only $22 \%$ of those were explored by nurses [17]. In addition, evidence showed that nurses only adequately responded to $25 \%$ of the emotional cues disclosed by head-neck cancer patients and their caregivers [14].

\section{Nurse-related Factors Influencing Managing Emotional Cues}

Nurses’ inadequate responses to emotional cues are closely linked to inhibitory behaviors 
including factual clarification, use of distancing strategies, and overt blocking. Factual clarification is defined as "continuing with the cue and clarifying evidence related to it; however, there is no further exploration of the identical cue". Distancing strategies include disrupting the flow of conversations or moving away from the emotional cue. By using overt blocking, the subject is changed to avoid the cue and content expressed by the patient [9]. These behaviors may reduce further disclosure or even block patients from revealing information or emotional cues [12]. The possible factors that lead to nurses' inadequate response include their lack of communication training, lack of confidence, and lack of time [17, 18]. In contrast, facilitating behaviors such as acknowledgement and exploration of the cues are considered adequate strategies that can facilitate patient emotional disclosure, enhance effective patient-provider communication, and allow more accurate information-gathering from patients [12].

Acknowledgement is defined as using checking, reflection, or empathy to respond to emotional cues without taking any further action. Exploring the cue means using a question or statement for eliciting information or clarifying the cue [9].

Other nurse-related barriers to cue responding may link to environmental factors (e.g., unbalanced nurse-to-patient ratio), task-oriented care models, or opinions about communication $[19,20]$. Although how nurses respond to patients' emotional cues is important and challenging, research that explores nurses' CRE thoroughly is scarce. Only one American study found no significant variables that can predict nurses' affective responsiveness behavior [15]. What affects nurses' CRE remains unclear, especially in the Chinese culture. The specific aims of this study were to (1) describe the standardized patients' (SP) emotional cues and nurses' cue-responding behaviors, (2) examine the correlation between the levels of emotional cues and the cueresponding behaviors, and (3) identify the predictive factors related to the CRE of Taiwanese nurses.

\section{Methods}

\section{Study Design}

An exploratory and predictive correlational study was conducted. A unidirectional sequential analysis was used to analyze the levels of SP's emotional cues and nurses' responding behaviors.

\section{Participants}

A convenience sample of registered nurses was recruited from two medical centers and three regional hospitals in the southern Taiwan. Although we recruited nurses in different units (e.g., oncology and palliative care units), eligible nurses must have had experience of caring for cancer patients. We excluded nurses who were in the three-month probationary period due to their unfamiliarity with working routines and environment. Among the 186 approached nurses, 120 nurses (65\%) agreed to participate. Upon confirming participation on the study, a researcher explained the overall study goal and process, including participating in a video-recorded, 15 minutes' conversation with a cancer patient. Nurses were not told that the primary focus is their response to emotions and the patient was simulating a cancer diagnosis. 


\section{Instruments}

Instruments used were a demographic questionnaire, the Medical Interview Aural Rating Scale, and the State-Trait Anxiety Inventory.

Medical Interview Aural Rating Scale (MIARS). The MIARS, developed by Heaven and Green, is widely used to examine patient-healthcare provider communication. The MIARS can be used to evaluate three communication domains. For the purpose of this study, two domains were assessed: patient's emotional disclosure (1 item) and nurse's response to emotional cues (5 items). Emotional disclosure was evaluated as cues that are expressions of emotion. Emotional cues were rated independently based on psychological depth (levels 1 to 3) of SP by two coders. A level-1 cue contains hints of emotion and implies some features of emotion. When a patient expresses a level-2 cue, he or she conveys a specific emotional state. A level-3 cue is an explicit or stronger level of emotion.

The nurse's response to emotional cues is coded into five cue-responding behaviors that belong to two categories: adequate response (i.e. exploration of the cue and acknowledgement of the cue) and inadequate response (i.e. factual clarification, use of distancing strategies, and overt blocking) [12]. These responses were defined by Heaven and Green (2001) and described in the background section. The MIARS has been applied to explore the emotional expression in cancer patients and patients receiving palliative care. In the current study, the inter-rater reliability coefficient $(\kappa)$ among two raters was 0.8 and intra-rater reliability was 0.78 , indicating the level of consistency and stability of the coders.

State-Trait Anxiety Inventory (STAI). Nurse anxiety was measured via the Chinese version of the STAI, modified by Chung and Lung [21] based on Spielberger's STAI. State anxiety measures the current anxiety status, including feelings of tension, worry, and arousal of the autonomic nervous system. Trait anxiety evaluates relatively stable aspects of anxiety that are related to personality. The STAI is a self-rated questionnaire using a 4-point Likert scale to assess two subscales: State Anxiety (20 items) and Trait Anxiety (20 items). The total score ranges from 20 to 80 . Higher score indicates a higher degree of anxiety. A total score of 20-39 represents low-level anxiety; a total score of 40-59 suggests middle-level anxiety; and 60-80 suggests a high-level anxiety. The STAI has been used in Taiwan with Cronbach's $\alpha$ of .90 and .86 for reliability and validity, respectively. In the current study, the Cronbach's $\alpha$ for State Anxiety Inventory and Trait Anxiety Inventory was .92 and .89, respectively.

\section{SP Training and Data Collection}

A standardized script was developed by the author (MFL) using actual case scenarios to demonstrate the physical and psychological manifestations of a newly diagnosed cancer patient. The script contains different levels of emotional cues as described by the MIARS. A professional director was hired to train 10 paid SPs regarding how to express different levels of emotional cues based on the scripts. All SPs received four 8-hour group training sessions. After training, each SP was evaluated by two researchers using the MIARS to ensure the consistency of emotional cue expression. Finally, six SPs were considered authentic and consistent, and they were randomly assigned to interact with the nurse during the 15-minute interview. All nurse- 
patient interactions occurred in a hospital private room. Participating nurses obtained written information prior to the interview about the patient's illness, chief complaint, and objectives of the upcoming nurse-patient interview (e.g., approaching a patient who received problematic test results). The nurses also completed demographic questionnaire and the STAI prior to the interview. All interviews were video recorded.

\section{Human Subjects}

Approval to conduct the study was obtained from each of the five participating hospitals. Written informed consent was obtained from all participants prior to the interviews.

\section{Data Analysis}

The interviews were coded independently by two master-prepared researchers who had received MIARS training. Based on the MIARS, each turn of conversation was coded as an analytic unit. The Observer ${ }^{\circledR}$ XT (Version 10; Noldus Information Technology, 2010) software was used to import the video-recordings and conduct coding analysis to enhance the validity of MIARS coding [22]. The frequencies of each behavior unit of patient and nurse were totaled for analysis. A unidirectional sequential analysis, adapted from the Multiple Episode Protocol Analysis Program developed by Erkens [23], was used to examine the correlation of patients' expression of emotional cues with nurses' responses to those cues. The relative kappa was calculated to reflect the degree of association between cues and responses $(-1 \leqq k \leqq 1)$. A higher kappa indicates a stronger correlation. To recognize the predictors of nurses' CRE, a cueresponding score was calculated [13]:

$$
\mathrm{CRE}=\frac{\text { adequate response }- \text { inadequate reponse }}{\text { total number of cue responses }}
$$

The CRE score ranges from 1 and -1, with a higher score representing higher competence of emotion-focused communication. SPSS 17.0 (IBM, Armonk, NY, 2008) was used to perform statistical analysis including descriptive statistics for describing demographic characteristics, Pearson's correlation, and a forward selection stepwise multiple regression for establishing a predictive model.

\section{Results}

\section{Demographics}

Of the 120 nurses who agreed to participate, 110 (91.6\%) completed the questionnaires and were included in the analysis. Participants worked at two medical centers ( $n=48,43.6 \%$ ) and three local hospitals $(n=62,56.4 \%)$ in southern Taiwan. All 110 were female. Their average age was $34(\mathrm{SD}=6.4)$ with more than half aged $23-33$. The average length of practice was 10.7 years ( $\mathrm{SD}=6.8)$. The majority $(\mathrm{n}=60,55 \%)$ had some college or higher education; however, most of them $(n=81,74 \%)$ had not received any communication training. Forty-three of the nurses (39\%) practiced in general medicine and surgery units, and 24 (22\%) practiced in oncology units. The average score of state anxiety was $50.1(\mathrm{SD}=10.4)$ with more than $85 \%$ (n 
= 93) of the participants' state anxiety equal to or higher than the middle level. The average score of trait anxiety was 46.0 (SD = 9.0) with 76\% $(\mathrm{n}=84)$ demonstrating middle- or high-level trait anxiety. Table 1 shows participants’ demographic characteristics.

\section{Emotional Cues and Cue-Responding Behavior}

The average was 23.4 emotional cues (95\% CI: 22.2-24.5) in each 15-minute interview, ranging from 10 to 42 emotional cues. Most emotional cues (46\%) were level 1 followed by level $2(38 \%)$ and level $3(16 \%)$. The majority $(n=2,139,83 \%)$ of nurses' cue-responding behavior were inadequate responses, including factual clarification, use of distancing strategies, and overt blocking. For the adequate cue-responding behaviors ( $\mathrm{n}=441,17 \%), 219$ responses (8.6\%) were exploration of the cue (e.g. What makes you feel sad?), and 222 (9\%) were acknowledgement of the cue (e.g. Are you at a loss because of your disease?) (Table 2).

\section{The Relationship Between Emotional Cues and Cue-Responding Behavior}

Based on unidirectional sequential analysis, participants used lots of distancing strategies ( $\kappa$ $=0.70,0.68,0.38$ ) regardless of the levels of emotional cues. On the other hand, the most frequent adequate response was exploring the cues $(\kappa=0.32)$ when patients' emotional cues were more implicit (i.e. level-1 cues). When patients' emotional cues were explicit (i.e. level-3 cues), participants tended to use more acknowledgement strategies $(\kappa=0.36)$ (Table 3$)$.

\section{Correlation of Demographics and CRE}

Based on Pearson's correlation analysis, the CRE positively correlated with prior communication training $(\mathrm{r}=0.39, \mathrm{p}=0.000)$, working at a palliative-care unit $(\mathrm{r}=0.35, \mathrm{p}=$ $0.000)$, and educational level $(r=0.23, p=0.016)$. On the other hand, the CRE negatively correlated with length of practice $(\mathrm{r}=-0.24, \mathrm{p}=0.009)$ and state-anxiety score $(\mathrm{r}=-0.23, \mathrm{p}=$ 0.014).

\section{Predictors of CRE}

The mean score of the independent variable, CRE, was -.064 (SD $=0.28$, ranging from -1 to 0.2 , CI: -0.6 to -0.7$)$. The predictors were selected to enter the regression model based on the level of their correlation with CRE. Prior formal communication training, practice unit (palliative-care unit vs. others), length of nursing practice, and level of education were significant predictors that together explained 36.3\% of the variance of CRE (Table 4).

\section{Discussion}

This observational study aimed to explore what influences nurses' CRE and the relationship between emotional cues and CRE. Although mean age, length of nursing practice, and educational level were slightly lower than those of nurses in the European and US studies [12, 20], the participants reflected a representative sample of nurses in Taiwan [24]. Overall, inadequate behaviors, especially distancing strategies, comprised the overwhelming responses of nurses. Although the use of distancing strategies decreased by $15 \%$ when more explicit level-2 or 
level-3 emotional cues were given, other negative strategies were still used to handle these cues. This phenomenon may be associated with nurses' inability to recognize subtle cues or nurses' decisions about the priorities of the communication. For example, nurses may prioritize physical care over psychological care [25].

It is noteworthy that Taiwanese nurses demonstrated more inadequate cue-responding behaviors than nurses in other countries. Compared to studies done in Hong Kong, UK, and Netherland which showed that about $50 \%$ of the nursing students' or nurses' cue-responding behaviors were distancing strategies $[13,19,20]$, participants in the current study used distancing strategies $81 \%$ of the time. Future studies are needed to explore what factors contribute to Taiwanese nurses' use of inappropriate strategies, including culture-specific reasons. Although addressing negative emotion is essential in patient-centered care, this practice may challenge cultural beliefs in maintaining communication harmony or "face." In some Chinese culture, "face" is conceptualized as an individual's contingent self-esteem [26]. In order to maintain communication harmony or "face" (e.g., sustaining a professional demeanor), nurses might suppress their own emotions and avoid patients' negative emotions [27]. Furthermore, evidence that adults from Asian and western cultures process socio-emotional cues differently has been identified recently [28]. Therefore, how cultural beliefs influence the nurses' responses to patients' expressions of emotion must be explored before conclusions are generated from crosscultural comparisons.

In the current study, four variables were found to be significant predictors of CRE: prior formal communication training, practice at a palliative unit, length of nursing practice, and educational level. Our finding with regard to prior formal communication training is similar to other studies that indicated nurses who have received this training can use communication skills more effectively. Communication-trained nurses were more competent to identify emotional distress, use open-ended questions, show empathy, be sensitive about patients' emotions, and respond and manage patients' emotional distress [29-31]. Unfortunately, only one-third of the nurses in this study had prior formal communication training. Hsiao and colleagues [32] reported that although more than $90 \%$ of the 38 nursing schools in Taiwan teach communication-related topics in basic nursing classes, the average teaching time is only 2.7 hours. Although no consensus yet exists on the appropriate amount of pre-license communication education in nursing, researchers have suggested that fewer than 40 hours of training may be insufficient [33]. Actually, more than $40 \%(n=44)$ of the nurses in our study expressed the need for communication training. The need to incorporate more structured communication training into nursing curriculum or continuing education programs is urgent.

Practice experience in a palliative-care unit also positively predicted CRE. Evidence indicated that palliative-care nurses were more skillful and comfortable at identifying patient suffering, evaluating psychological needs, and discussing emotional issues [34, 35]. Providing cross-training opportunities for nurses in palliative-care units may enhance their CRE. On the other hand, our results showed that length of experience negatively predicted CRE. A Polish study $(\mathrm{n}=108)$ illustrated similar findings by demonstrating that nurses had poor communication ability compared to nursing students. The researchers suspected that nurses' decreased communication competence may come from work stress [36]. A study in Israel also revealed a negative relationship between nursing burnout and nurses' self-efficacy in discussing special 
subjects with patients [34]. Therefore, more practice experience may not guarantee greater CRE. In addition to communication training, addressing nurses' emotional needs may be equally important in promoting nurses’ CRE.

It is not surprising to find that educational level was a positive predictor of CRE. Taiwanese nurses with a higher educational level are more competent than nurses with a lower educational level in terms of overall nursing care and are more sensitive to emotions [37]. One assumption is that the general learning goals of a college education stimulate critical thinking, enhance teamwork ability, and focus on independent practice. However, nurses with a technical education comprise the majority of the nursing workforce in Taiwan. Among the 145,172 practicing Taiwanese nurses (January, 2014), 47\% have a technical 3-year degree as their highest nursing degree [38].

Finally, nurses' state anxiety negatively correlated to CRE, but not their trait anxiety. That is, nurses' communication-related anxiety stems from their current feelings, rather than from relatively stable aspects of anxiety related to personality. Nurses' state anxiety related to communication can be affected by several factors, such as encountering difficult subjects and worrying about one's own emotions [18]. As up to 85\% of the participating nurse reported middle-level anxiety, it may due to their awareness of being recorded and studied $[12,15]$ and the designed scenario which was related to difficult subjects. Addressing nurses’ personal stress related to communication is important because nurses who are more stressed feel less confident in responding to emotional cues [39].

Our study has some limitations, including the use of convenience sampling and the Hawthorne effect related to video-recording. The results may not be applicable to male nurses who have different communication styles [15, 37]. Although SPs were trained to reflect designed emotional cues, their performance may have been affected by the nurses' responses, especially when the responses exceeded the scope of the standardized script.

\section{Conclusions}

To our knowledge, this is the first study examining Taiwanese nurses’ CRE. Analyzing nurses' CRE is an innovative micro-level method to interpret nurse-patient communication [12]. Taiwanese nurses used more inadequate responses to all levels of emotional cues than nurses in other countries. Future study is needed to explore possible reasons, such as cultural differences. Significant predictors of CRE were identified: prior formal communication training, practice unit, length of nursing practice, and educational level. Researchers and educators must consider how education and working environments affect nurses’ CRE. 


\section{References}

1. WHO. GLOCOBAN: Estimated Incidence. Mortality and Prevalence Worldwide in 2012. Retrieved April 16, 2015 from: http:/globocan.iarc.fr/Pages/fact_sheets_cancer.aspx,

2. Dunn J, Ng SK, Holland J, et al. Trajectories of psychological distress after colorectal cancer. Psycho-Oncology 2013;22:1759-1765. DOI: 10.1002/pon.3210

3. Zabora J, Brintzenhofeszoc K, Curbow B, Hooker C, Piantadosi S. The prevalence of psychological distress by cancer site. Psycho-Oncology 2001;10:19-28.

4. Chambers SK, Hyde MK, Ip DF, Dunn JC, Gardiner RA. Systematic review of research into the psychological aspects of prostate cancer in Asia: what do we know? Asian Pac J Cancer Prev 2013;14:2621-2626.

5. Chen ML, Chang HK, Yeh CH. Anxiety and depression in Taiwanese cancer patients with and without pain. $J$ Adv Nurs 2000;32:944-951.

6. Chen SC, Liao CT, Lin CC, Chang JT, Lai YH. Distress and care needs in newly diagnosed oral cavity cancer patients receiving surgery. Oral Oncol 2009;45:815-820. DOI: 10.1016/j.oraloncology.2009.01.001

7. Liao YC, Liao WY, Shun SC, et al. Symptoms, psychological distress, and supportive care needs in lung cancer patients. Support Care Cancer 2011;19:1743-1751. DOI: 10.1007/s00520-010-1014-7

8. Sheldon LK, Blonquist TM, Hilaire DM, Hong F, Berry DL. Patient cues and symptoms of psychosocial distress: what predicts assessment and treatment of distress by oncology clinicians? Psycho-Oncology 2015;24:1020-1027. DOI: 10.1002/pon.3689

9. Heaven C, Green C. Medical Interview Aural Rating Scale. 2001, Christie Hospital, Stanley House: Psychological Medicine Group: Manchester, UK, 2001.

10. Heyn L, Finset A, Ruland CM. Talking about feelings and worries in cancer consultations: the effects of an interactive tailored symptom assessment on source, explicitness, and timing of emotional cues and concerns. Cancer Nurs 2013;36:E20-E30. DOI: 10.1097/NCC.0b013e318254af66

11. Jansen J, van Weert JC, de Groot J, et al. Emotional and informational patient cues: the impact of nurses' responses on recall. Patient Educ Couns 2010;79:218-224. DOI: 10.1016/j.pec.2009.10.010

12. Uitterhoeve R, de Leeuw J, Bensing J, et al. Cue-responding behaviours of oncology nurses in video-simulated interviews. J Adv Nurs 2008;61:71-80. DOI: 10.1111/j.13652648.2007.04467.x

13. Uitterhoeve R, Bensing J, Dilven E, et al. Nurse-patient communication in cancer care: does responding to patient's cues predict patient satisfaction with communication. Psycho-Oncology 2009;18:1060-1068. DOI: 10.1002/pon.1434

14. de Leeuw J, Prins JB, Uitterhoeve R, et al. Nurse-patient communication in follow-up consultations after head and neck cancer treatment. Cancer Nurs 2014;37:E1-E9. DOI: 10.1097/NCC.0b013e318288d3f3

15. Sheldon LK, Ellington L, Barrett R, et al. Nurse responsiveness to cancer patient expressions of emotion. Patient Educ Couns 2009;76:63-70. DOI:

10.1016/j.pec.2008.11.010

16. Butow PN, Brown RF, Cogar S, Tattersall MH, Dunn SM. Oncologists' reactions to cancer patients' verbal cues. Psycho-Oncology 2002;11:471-458.

17. Sheldon LK, Hilaire D, Berry DL. Provider verbal responses to patient distress cues 
during ambulatory oncology visits. Oncol Nurs Forum 2011;38:369-375. DOI: 10.1188/11.onf.369-375

18. Heaven C, Maguire P, Green C. A patient-centred approach to defining and assessing interviewing competency. Epidemiol Psichiatr Soc 2003;12:86-91.

19. Chan EA. Cue-responding during simulated routine nursing care: a mixed method study. Nurse Educ Today 2014;34:1057-1061. DOI: 10.1016/j.nedt.2014.02.010

20. Heaven C, Clegg J, Maguire P. Transfer of communication skills training from workshop to workplace: the impact of clinical supervision. Patient Educ Couns 2006;60:313-325. DOI: $10.1016 /$ j.pec.2005.08.008

21. Chung SC, Lung CF. Modifying the state-trait anxiety inventory. Psychological Testing 1984;31:27-36.

22. Fletcher I, Heaven C, Maguire P, Dunn G. A comparison of traditional vs. computerised methods of rating communications skills In International Conference on Communication in Healthcare. University of Bruges: Bruges, 2004.

23. Erkens G. Multiple Episode Protocol Analysis (MEPA), 2003. Available from: http://edugate.fss.uu.nl/mepa/. [2009]

24. Chang CC, Lin LM, Chen IH, Kang CM, Chang WY. Perceptions and experiences of nurse preceptors regarding their training courses: a mixed method study. Nurse Educ Today 2015;35:220-226. DOI: 10.1016/j.nedt.2014.08.002

25. Lin YT. Therapeutic discourse: on the intersubjective nurse-patient relationship. $J$ Nurs 2008;55:14-19.

26. Hwang KK. Moral face and social face: contingent self-esteem in Confucian society. . Int J Psychol 2006;41:276-281.

27. Vilelas JM, Diogo PM. Emotional labor in nursing praxis. Rev Gaucha Enferm 2014;35:145-149.

28. Liu P, Rigoulot S, Pell MD. Culture modulates the brain response to human expressions of emotion: electrophysiological evidence. Neuropsychologia 2015;67:1-13.

29. Fukui S, Ogawa K, Ohtsuka M, Fukui N. Effect of communication skills training on nurses' detection of patients' distress and related factors after cancer diagnosis: a randomized study. Psychooncology 2009;18:1156-1164. DOI: 10.1002/pon.1429

30. Meyer EC, Sellers DE, Browning DM, et al. Difficult conversations: improving communication skills and relational abilities in health care. Pediatr Crit Care Med 2009;10:352-359. DOI: 10.1097/PCC.0b013e3181a3183a

31. Moore PM, Rivera Mercado S, Grez Artigues M, Lawrie TA. Communication skills training for healthcare professionals working with people who have cancer. Cochrane Database Syst Rev 2013;3:Cd003751. DOI: 10.1002/14651858.CD003751.pub3

32. Hsiao YC, Wu LY, Cheng HY, Liu PH. Reflection on student nurses' communication competence. Chang Gung Nurs 2012;23:464-475.

33. Chant S, Tim, Randle J, Russell G, Webb C. Communication skills training in healthcare: a review of the literature. Nurse Educ Today 2002;22:189-202. DOI: 10.1054/nedt.2001.0690

34. Emold C, Schneider N, Meller I, Yagil Y. Communication skills, working environment and burnout among oncology nurses. Eur J Oncol Nurs 2011;15:358-363. DOI: 10.1016/j.ejon.2010.08.001

35. Ronaldson S, Hayes L, Aggar C, Green J, Carey M. Spirituality and spiritual caring: nurses' perspectives and practice in palliative and acute care environments. J Clin Nurs 
2012;21:2126-2135. DOI: 10.1111/j.1365-2702.2012.04180.x

36. Wloszczak-Szubzda A, Jarosz MJ. Professional communication competences of nurses. Ann Agric Environ Med 2012;19:601-607.

37. Hsieh CJ, Chang HW, Chou YR, Chang C. The relationship between emotional intelligence and burnout in nurses. New Taipei Journal of Nursing 2008;10:11-24.

38. Teng SW. Nursing manpower and solutions in Taiwan. J Nurs 2014;61:5-12. DOI: 10.6224/jn.61.2.5

39. Loureiro EM, Severo M, Bettencourt P, Ferreira MA. Attitudes and anxiety levels of medical students towards the acquisition of competencies in communication skills. Patient Educ Couns 2011;85:E272-E277. DOI: 10.1016/j.pec.2011.07.005 
Table 1. Participant Demographics $(\mathrm{N}=110)$

\begin{tabular}{|c|c|c|c|c|}
\hline Characteristic & $n$ & $(\%)$ & Mean & $(\mathrm{SD})$ \\
\hline Age & & & 34 & $(6.4)$ \\
\hline Years of nursing practice & & & 10.7 & $(6.8)$ \\
\hline $0-7$ & 38 & (35) & & \\
\hline $8-13$ & 39 & (35) & & \\
\hline$>14$ & 33 & (30) & & \\
\hline \multicolumn{5}{|l|}{ Level of education } \\
\hline Junior college (Diploma) & 8 & $(7)$ & & \\
\hline Technical college (Diploma) & 42 & (38) & & \\
\hline University (Bachelor) & 60 & (55) & & \\
\hline \multicolumn{5}{|l|}{ Practice units } \\
\hline Medical/Surgical & 50 & $(45)$ & & \\
\hline Oncology & 36 & (33) & & \\
\hline Palliative & 15 & (14) & & \\
\hline Emergency room/ Intensive care unit & 9 & $(8)$ & & \\
\hline \multicolumn{5}{|l|}{ Prior formal communication training } \\
\hline Yes & 29 & (26) & & \\
\hline No & 81 & (74) & & \\
\hline State Anxiety & & & 50.1 & $(10.4)$ \\
\hline Mild (20-39) & 17 & (16) & & \\
\hline Moderate (40-59) & 77 & (70) & & \\
\hline Severe (60-80) & 16 & (15) & & \\
\hline Trait Anxiety & & & 46.0 & $(9.0)$ \\
\hline Mild (20-39) & 26 & (24) & & \\
\hline Moderate (40-59) & 76 & (69) & & \\
\hline Severe (60-80) & 8 & (7) & & \\
\hline
\end{tabular}


Table 2. Patients' Emotional Cues and Nurses' Responding Behaviors

\begin{tabular}{lrcccc}
\hline Behavioral elements & \multicolumn{2}{c}{ Frequencies } & Mean per & 95\% CI & SD \\
& $\mathrm{n}$ & $(\%)$ & conversation & & \\
\hline Patient cues & & & & & \\
$\quad$ Level 1 & 1231 & $(46)$ & 10.90 & $10.00-11.80$ & 4.90 \\
$\quad$ Level 2 & 1020 & $(38)$ & 8.80 & $8.20-9.40$ & 3.30 \\
$\quad$ Level 3 & 414 & $(16)$ & 3.70 & $3.40-4.00$ & 1.60 \\
Nurse cue-responding behaviors & & & & & \\
Adequate response & 441 & $(17)$ & 4.07 & $3.40-4.80$ & 3.85 \\
$\quad$ Exploration of the cue & 219 & $(8.6)$ & 2.10 & $1.60-2.50$ & 2.30 \\
$\quad$ Acknowledgement of the cue & 222 & $(9)$ & 2.00 & $1.50-2.40$ & 2.40 \\
Inadequate response & 2139 & $(83)$ & 18.70 & $17.50-20.00$ & 6.06 \\
$\quad$ Factual clarification & 23 & $(1)$ & 0.20 & $0.10-0.30$ & 0.40 \\
$\quad$ Use of distancing strategies & 2105 & $(81)$ & 18.40 & $17.30-19.60$ & 6.20 \\
$\quad$ Overt blocking & 11 & $(0.4)$ & 0.10 & $0.00-0.20$ & 0.40 \\
\hline
\end{tabular}


Table 3. Nurses’ Adequate and Inadequate Responses by Levels of Patients' Emotional Cue and Their Relationships as Reflected by Kappa

\begin{tabular}{|c|c|c|c|c|c|c|c|c|c|c|}
\hline \multirow{3}{*}{$\begin{array}{c}\text { Cue } \\
\text { Level } \\
\end{array}$} & \multicolumn{4}{|c|}{ Adequate Response } & \multicolumn{6}{|c|}{ Inadequate Response } \\
\hline & \multicolumn{2}{|c|}{ Exploration of cue } & \multicolumn{2}{|c|}{$\begin{array}{l}\text { Acknowledgement } \\
\text { of cue }\end{array}$} & \multicolumn{2}{|c|}{ Factual clarification } & \multicolumn{2}{|c|}{ Distancing strategies } & \multicolumn{2}{|c|}{ Overt blocking } \\
\hline & $\mathrm{N}(\%)$ & Kappa & $\mathrm{N}(\%)$ & Kappa & $\mathrm{N}(\%)$ & Kappa & $\mathrm{N}(\%)$ & Kappa & $\mathrm{N}(\%)$ & Kappa \\
\hline 1 & $104(8.6)$ & 0.32 & $65(5.4)$ & 0.09 & $10(0.8)$ & 0.27 & $1011(84.5)$ & 0.70 & $6(0.5)$ & 0.41 \\
\hline 2 & 84(8.5) & 0.24 & $64(6.5)$ & 0.12 & $11(1.1)$ & 0.36 & 821(83.4) & 0.68 & $4(0.4)$ & 0.22 \\
\hline 3 & $30(7.8)$ & 0.07 & $91(24)$ & 0.36 & $1(0.2)$ & -0.47 & $257(67.6)$ & 0.38 & $1(0.2)$ & 0.02 \\
\hline
\end{tabular}


Table 4. Regression Analysis for Variables Predicting Nurses' Competence of Emotional Cue-Responding (Adjusted $R^{2}=36.3 \%$ )

\begin{tabular}{|c|c|c|c|c|}
\hline Independent variable & $ß$ & $95 \% \mathrm{CI}$ & $\mathrm{t}$ & $p$ \\
\hline Prior formal communication training & 0.369 & $0.138 / 0.332$ & 4.820 & .000 \\
\hline Practice units (Hospice or other) & 0.306 & $0.127 / \quad 0.373$ & 4.023 & .000 \\
\hline Years of nursing practice & -0.305 & $-0.019 / \quad-0.006$ & -3.982 & .000 \\
\hline Level of education & 0.216 & $0.029 / \quad 0.165$ & 2.828 & .006 \\
\hline State anxiety & -0.144 & $-0.008 / \quad 0.000$ & -1.889 & .062 \\
\hline
\end{tabular}

